\title{
An Experience of Landiolol Use for an Advanced Heart Failure Patient With Severe Hypotension
}

\author{
Daisuke NitTa, ${ }^{1}$ MD, Koichiro Kinugawa, ${ }^{2}$ MD, Teruhiko Imamura, ${ }^{2}$ MD, \\ Miyoko Endo, ${ }^{3}$ RN, Eisuke AmIYA, ${ }^{1}$ MD, Toshiro InABA, ${ }^{1}$ MD, Hisataka MakI, ${ }^{1}$ MD, \\ Masaru Hatano, ${ }^{1} \mathrm{MD}$, and Issei Komuro, ${ }^{1} \mathrm{MD}$
}

\begin{abstract}
SUMMARY
Tachyarrhythmias such as atrial fibrillation (AF) or atrial flutter (AFL) sometimes invoke life-threatening collapse of hemodynamics in patients with severe heart failure. Recently, landiolol, an ultra-short acting $\beta 1$-selective antagonist, has been reported to be safe and useful for the treatment of supraventricular tachyarrhythmias with reduced left ventricular function. Here we report a case of advanced heart failure with severe hypotension who was treated successfully by landiolol for rapid AF. The patient was a 20-year old male with dilated cardiomyopathy. He presented with low output syndrome in spite of optimal medical therapy and was referred to our department to consider ventricular assist device implantation and heart transplantation. Soon after admission, he developed rapid atrial fibrillation at 180 beats per minute (bpm) followed by severe hypotension and liver enzyme elevation. Low dose landiolol at $2 \mu \mathrm{g} / \mathrm{kg} /$ minute was started because digoxin was not effective. After landiolol administration, his heart rate decreased to $110 \mathrm{bpm}$, and finally returned to sinus rhythm without hemodynamic deterioration. Intra-aortic balloon pumping was inserted soon after sinus recovery and he was discharged successfully with an implantable left ventricular assist device. (Int Heart J 2015; 56: 564-567)
\end{abstract}

Key words: Atrial fibrillation, Stage D heart failure, Negative chronotropic effect, Ventricular assist device

$\mathrm{I}$ is well known that $\beta$ blocker treatment is crucial for the improvement of prognosis among patients with systolic heart failure. ${ }^{1-3)}$ However, the usage of $\beta$ blockers during the acute phase of decompensated heart failure is still a matter of debate. Propranolol, a classic intravenous form of $\beta$ blocker, has been used widely for perioperative arrhythmias. However, there are concerns about bronchospasm due to the non-selectivity of $\beta$ adrenergic receptors. Their long half-life period may also result in the prolongation of adverse events if they occur.

Landiolol was developed in Japan and introduced as an ultra-short acting intravenous $\beta$ blocker. It has very high selectivity for $\beta 1$ receptors and is rapidly degraded by carboxylesterase and cholinesterase in the liver and blood, respectively. Recently, many papers have reported on the benefits of landiolol for the control of perioperative tachyarrhythmias. ${ }^{4)}$ However, the indication of landiolol has been limited in patients with preserved ejection fraction, and only digoxin is indicated to control heart rate in patients with severe left ventricular (LV) dysfunction.

The J-Land study (Japanese landiolol versus digoxin study) demonstrated that landiolol achieved faster control of heart rate among AF/AFL patients with LV dysfunction compared with digoxin. ${ }^{5,6)}$ The safety profiles were comparable between the two drugs. In the J-Land study, however, patients with severe LV dysfunction (ejection fraction: $\mathrm{EF}<25 \%$ ) or severe hypotension (systolic blood pressure $<90 \mathrm{mmHg}$ ) were excluded and there has been limited experience of landiolol among such patients. We here report an experience of landiolol use for an advanced heart failure patient with severe hypotension.

\section{Case Report}

The patient was a 20-year old male with dilated cardiomyopathy and tethering mitral regurgitation (MR) from left ventricular enlargement. A cardiac murmur was discovered at 6-years of age and he was diagnosed as having dilated cardiomyopathy from endomyocardial biopsy. Oral medication therapy including enalapril, furosemide and spironolactone was started. When he was 15 years-old, the left ventricular diastolic diameter (LVDd) was $57 \mathrm{~mm}$ with moderate to severe MR. A small dose of carvedilol was initiated, but he still suffered from dyspnea on effort and fatigue. At 19 years-old, his LV was markedly enlarged (LVDd $75 \mathrm{~mm}$ ) with an elevated plasma level of B-type natriuretic peptide (BNP, $121 \mathrm{pg} / \mathrm{mL}$ ). Subsequently he was admitted to hospital because of the worsening symptom and received intensive medical therapy, but no improvement was observed. He was referred to our department for further treatment. His blood pressure and heart rate were

From the Departments of ${ }^{1}$ Cardiovascular Medicine, ${ }^{2}$ Therapeutic Strategy for Heart Failure, and ${ }^{3}$ Organ Transplantation, Graduate School of Medicine, The University of Tokyo, Tokyo, Japan.

Address for correspondence: Koichiro Kinugawa, MD, Department of Therapeutic Strategy for Heart Failure, Graduate School of Medicine, The University of Tokyo, 7-3-1 Hongo, Bunkyo-ku, Tokyo 113-8655, Japan. E-mail: kinugawa-tky@umin.ac.jp

Received for publication March 2, 2015. Revised and accepted March 18, 2015.

Released in advance online on J-STAGE September 11, 2015.

All rights reserved by the International Heart Journal Association. 
92/60 mmHg and 106 beats per minute (bpm) (sinus rhythm), respectively, on admission. Blood tests revealed no abnormal values, including creatinine and liver enzyme. The only exception was an elevated plasma level of BNP (412 pg/mL). From transthoracic echocardiography, the LVDd, left atrial diameter, and EF were $79 \mathrm{~mm}, 70 \mathrm{~mm}$, and $31 \%$, respectively. Medication consisted of $40 \mathrm{mg}$ furosemide, $50 \mathrm{mg}$ spironolactone, 10 mg enalapril, and $20 \mathrm{mg}$ carvedilol. We soon started continuous infusion of dobutamine at $2 \mu \mathrm{g} / \mathrm{kg} /$ minute. Though his symptoms improved slightly, AF occurred suddenly on day 13 (Figure 1). The rate of AF was 140-180 bpm and was easily accelerated by exertion. He complained of palpitations and nausea because of low output syndrome. Due to the rapid AF, systolic blood pressure fell to $70 \mathrm{mmHg}$ and oliguria was observed. Moreover, total bilirubin was elevated $(1.6 \mathrm{mg} / \mathrm{dL})$. Even though we injected $0.25 \mathrm{mg}$ digoxin intravenously at first, the rate several hours after the injection was still approximately 150-180 bpm. Because intravenous digoxin infusion had no effect on controlling his heart rate, we decided to administer a low dose of landiolol $(2 \mu \mathrm{g} / \mathrm{kg} /$ minute). Two hours after landiolol administration, the heart rate at rest was significantly decreased at 120-130 bpm without deterioration of blood pressure (systolic blood pressure was approximately 70 $\mathrm{mmHg}$ ). Because his heart rate was easily accelerated by exertion or diet to $140-150 \mathrm{bpm}$, we raised the landiolol dose to 4 $\mu \mathrm{g} / \mathrm{kg} /$ minute the next day. Subsequently, the heart rate at rest diminished to $110 \mathrm{bpm}$. Because his heart rate was still 130$140 \mathrm{bpm}$ at exertion, we titrated the dose of landiolol with careful attention to hemodynamics, finally up to $8 \mu \mathrm{g} / \mathrm{kg} /$ minute on day 16. At that time his heart rate was $110-120 \mathrm{bpm}$ at even exertion and the systolic blood pressure was maintained at approximately $70 \mathrm{mmHg}$. We aimed for $110 \mathrm{bpm}$ based on the result of the RACE II study. ${ }^{7)}$ The heart rate of AF was stabilized at approximately 100-110 bpm and total bilirubin decreased. We administered $200 \mathrm{mg} /$ day amiodarone orally on day 16 to reinforce the heart rate control and antici- pated a rhythm control effect. Right heart catheterization was performed on day 19. Mean right atrial pressure was 10 $\mathrm{mmHg}$, mean pulmonary capillary wedge pressure was 21 $\mathrm{mmHg}$, mean pulmonary artery pressure was $28 \mathrm{mmHg}$, cardiac index was $2.56 \mathrm{~L} /$ minute $/ \mathrm{m}^{2}$ (thermo-dilution method), and mean systemic blood pressure was $53 \mathrm{mmHg}$. Because the cardiac index was preserved under rate control and remodeling of the left atrium was severe, we did not perform direct current defibrillation. On day 20, his rhythm recovered to sinus rhythm, although the BNP level was still high $(780 \mathrm{pg} / \mathrm{mL})$. As he regained regular rhythm, we inserted an intra-aortic balloon pumping (IABP) on day 22 because the mean systemic blood pressure was under $60 \mathrm{mmHg}$ and oliguria was still present. Landiolol was discontinued after sinus conversion. On the same day, our institutional review board approved a heart transplantation and an implantable left ventricular assist device (LVAD). On day 26, he successfully received an implantable LVAD (HeartMate II ${ }^{\circledR}$, Thoratec Corporation, Pleasanton, CA, USA) (Figure 2). He was discharged on day 70 without any major complications.

\section{Discussion}

Atrial fibrillation (AF) and atrial flutter (AFL) are common arrhythmias in patients with severe heart failure, which sometimes aggravate hemodynamics. ${ }^{8)}$ The prevalence of AF increases with progression of NYHA (New York Heart Association) class and was reported that it approaches 50\% at most in class IV. ${ }^{9)}$ An inappropriate rapid ventricular response with $\mathrm{AF} /$ AFL causes the elevation of myocardial $\mathrm{O}_{2}$ demand and insufficient diastolic filling pressure that results in further deterioration of heart failure. ${ }^{10)}$ Depressed cardiac function sometimes requires IABP insertion but an irregular ventricular response often poses a barrier to its effective hemodynamic support. A large meta-analysis concluded that $\mathrm{AF}$ is associated with worse
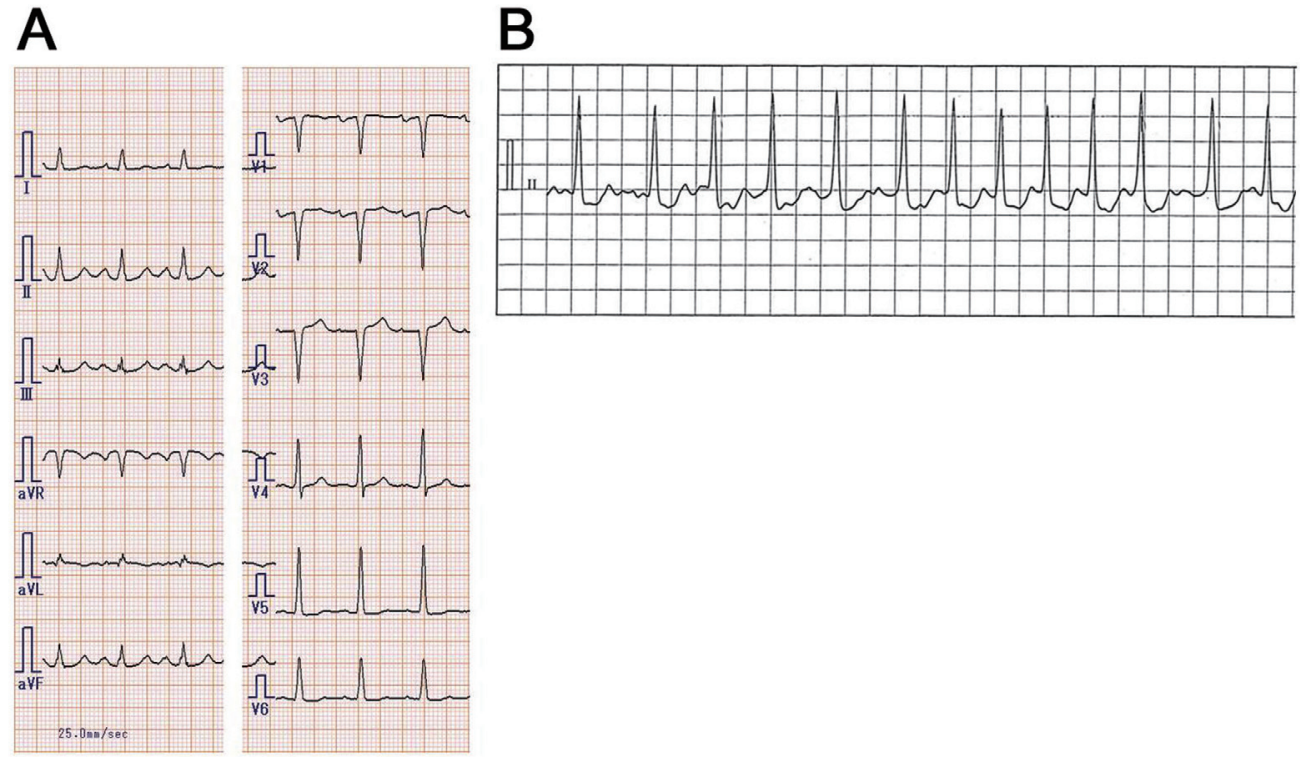

Figure 1. A: Electrocardiogram at sinus rhythm. Heart rate was $106 \mathrm{bpm}$. B: Monitor electrocardiogram at AF. R wave was irregular and heart rate was $140-180 \mathrm{bpm}$. Bpm indicates beats per minute. 


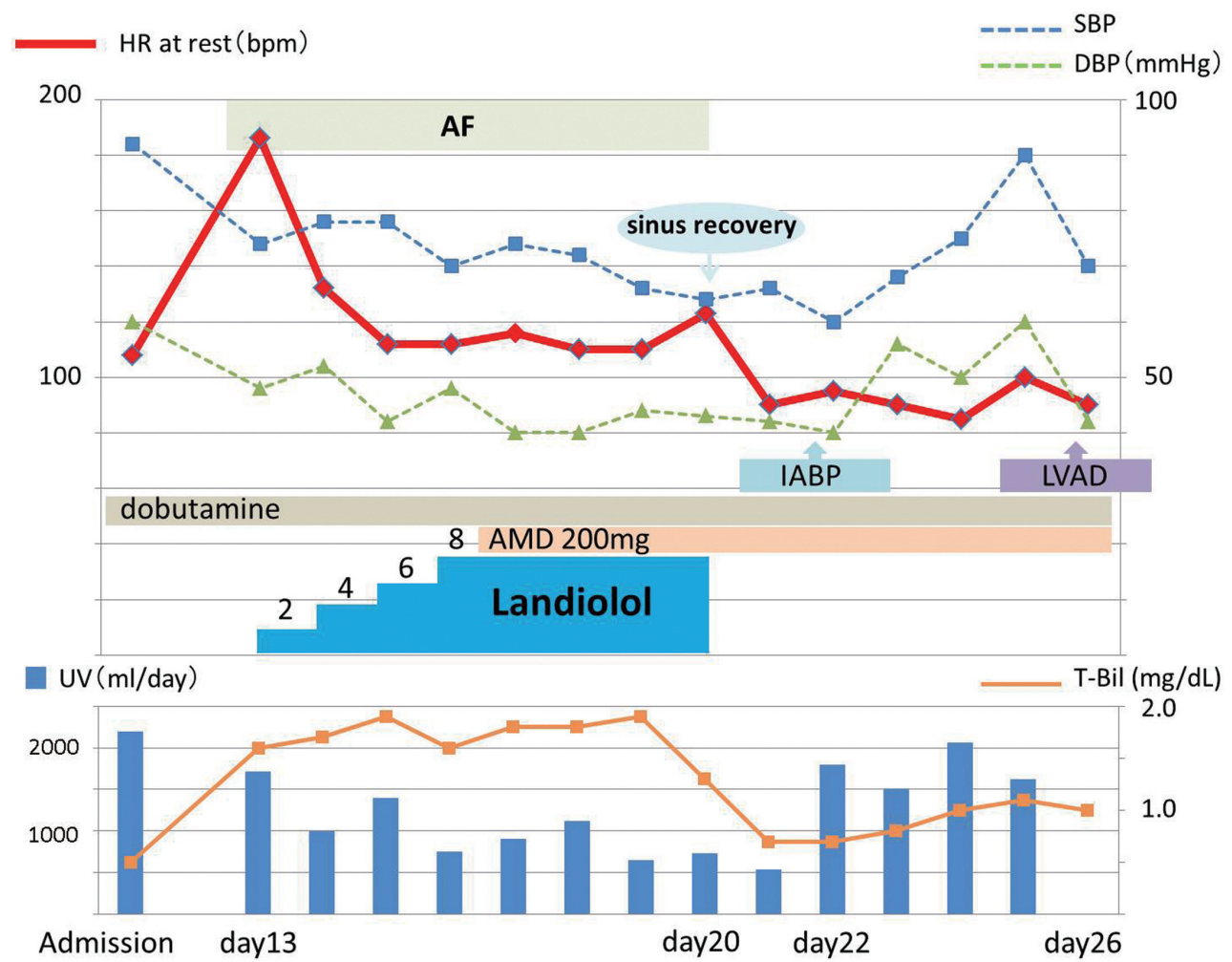

Figure 2. Time course of the present case after AF development. At first, AF heart rate reached $180 \mathrm{bpm}$, though it was gradually diminished by intravenous landiolol administration without significant deterioration of hypotension. After administration of amiodarone in addition to sufficient rate control, sinus recovery was obtained and IABP was inserted afterward. AF indicates atrial fibrillation; HR, heart rate; SBP, systolic blood pressure; DBP, diastolic blood pressure; AMD, amiodarone; IABP, intra-aortic balloon pumping; LVAD, left ventricular assist device ; UV, urine volume; and T-bil, total bilirubin. The unit of landiolol dose is $\mu \mathrm{g} / \mathrm{kg} /$ minute

mortality among heart failure patients with reduced LV function (odds ratio 1.49). ${ }^{11)}$

There are two strategies for the management of AF/AFL, ie, rate control and rhythm control. ${ }^{12)}$ In patients with severe heart failure, a rhythm control strategy using antiarrhythmic drugs often results in failure because of long-term remodeling of the left atrium/ventricle. In addition, the proarrhythmic effects of these drugs may be another concern. Furthermore, ablation carries high periprocedural risk of worsening heart failure in such unstable patients as our case. ${ }^{13)}$ Moreover, direct current defibrillation is not preferable because of further myocardial damage by the defibrillation itself, sedation-induced hypotension, or hemodynamic deterioration owing to sinus arrest after rapid AF termination.

Thus, rate control is commonly selected in advanced heart failure patients with AF. The guideline of the American Heart Association and the European Society of Cardiology recommends digitalis and amiodarone for acute rate control of $\mathrm{AF}$ in patients with heart failure and hypotension. ${ }^{14)}$ Digitalis has conventionally been used in heart failure patients because of its lack of a negative inotropic effect, although it takes several hours to attain sufficient action and some studies have warned that digitalis use was associated with elevated risk for all-cause mortality in patients with heart failure. ${ }^{15)}$ Amiodarone is available not only for rhythm control but also rate control, ${ }^{16)}$ whereas the half-life period is very long and it is difficult to adjust its dose within an appropriate range. Another intravenous $\beta$ blocker, esmolol, also has high $\beta 1$ selectivity and a short half-life period, ${ }^{17,18)}$ and its strong negative inotropic effect sometimes results in severe hypotension. ${ }^{19}$

Because patients with severe hypotension (systolic blood pressure $<90 \mathrm{mmHg}$ ) were excluded in the J-land study, there is little evidence about its use in patients like the present case. As his hemodynamics were deteriorating even under intravenous inotropic support, we concluded that LVAD should be indicated for him immediately (INTERMACS profile 2). ${ }^{20)}$ To obtain Japanese insurance reimbursement, we must receive approval from the institutional review board for the heart transplantation before implantable LVAD therapy. Appropriate rate control by landiolol, sinus conversion by amiodarone, and IABP insertion were all necessary in order to buy sufficient time for the examinations for heart transplant registration in this case.

Another important point is that landiolol was able to exhibit sufficient negative chronotropic effects when used simultaneously with dobutamine. Oral $\beta$ blockers, even bisoprolol which has high selectivity to $\beta 1$ receptors, are now routinely used with dobutamine. This strategy is practically useful for titrating $\beta$ blockers without worsening heart failure. Similarly, intravenous $\beta$ blockers can be used with dobutamine and may result in favorable results in terms of $\mathrm{AF}$ rate control. However, further investigations are necessary to determine the clinical 
significance and to clarify the mechanism of concomitant usage.

In conclusion, we successfully bridged an advanced heart failure patient with rapid AF and severe hypotension to implantable LVAD therapy by the careful introduction of landiolol. Although there have been few reports in this area, landiolol may be promising for AF rate control even in severe heart failure patients who require inotropic infusion.

\section{REFERENCES}

1. Effect of metoprolol CR/XL in chronic heart failure: Metoprolol CR/XL Randomised Intervention Trial in Congestive Heart Failure (MERIT-HF). Lancet 1999; 353: 2001-7.

2. The Cardiac Insufficiency Bisoprolol Study II (CIBIS-II): a randomised trial. Lancet 1999; 353: 9-13.

3. Packer M, Fowler MB, Roecker EB, et al. Effect of carvedilol on the morbidity of patients with severe chronic heart failure: results of the carvedilol prospective randomized cumulative survival (COPERNICUS) study. Circulation 2002; 106: 2194-9.

4. Sakamoto A, Hamasaki T, Kitakaze M. Perioperative landiolol administration reduces atrial fibrillation after cardiac surgery: A meta-analysis of randomized controlled trials. Adv Ther 2014; 31 : 440-50.

5. Nagai R, Kinugawa $\mathrm{K}$, Inoue $\mathrm{H}$, et al. Urgent management of rapid heart rate in patients with atrial fibrillation/flutter and left ventricular dysfunction: comparison of the ultra-short-acting beta1selective blocker landiolol with digoxin (J-Land Study). Circ J 2013; 77: 908-16.

6. Kinugawa $\mathrm{K}$, Nagai $\mathrm{R}$, Inoue $\mathrm{H}$, et al. Impacts of patient characteristics on the effectiveness of landiolol in AF/AFL patients complicated with LV dysfunction: Subgroup analysis of the J-Land study. Adv Ther 2014; 31: 426-39.

7. Van Gelder IC, Groenveld HF, Crijns HJ, et al. Lenient versus strict rate control in patients with atrial fibrillation. N Engl J Med 2010; 362: 1363-73.

8. Clark DM, Plumb VJ, Epstein AE, Kay GN. Hemodynamic effects of an irregular sequence of ventricular cycle lengths during atrial fibrillation. J Am Coll Cardiol 1997; 30: 1039-45.

9. Maisel WH, Stevenson LW. Atrial fibrillation in heart failure: epidemiology, pathophysiology, and rationale for therapy. Am J Cardiol 2003; 91: 2D-8D. (Review)

10. Wang TJ, Larson MG, Levy D, et al. Temporal relations of atrial fibrillation and congestive heart failure and their joint influence on mortality: the Framingham Heart Study. Circulation 2003; 107: 2920-5.

11. Mamas MA, Caldwell JC, Chacko S, Garratt CJ, Fath-Ordoubadi F, Neyses L. A meta-analysis of the prognostic significance of atrial fibrillation in chronic heart failure. Eur J Heart Fail 2009; 11: 67683.

12. Wyse DG, Waldo AL, DiMarco JP, et al. A comparison of rate control and rhythm control in patients with atrial fibrillation. $\mathrm{N}$ Engl J Med 2002; 347: 1825-33.

13. Sairaku A, Yoshida Y, Hirayama H, Nakano Y, Kihara Y. Impact of pulmonary vein isolation on fractionated atrial potentials and ganglionated plexi in patients with persistent atrial fibrillation. Int Heart J 2014; 55: 494-8.

14. Fuster V, Ryden LE, Cannom DS, et al. 2011 ACCF/AHA/HRS focused updates incorporated into the ACC/AHA/ESC 2006 Guidelines for the management of patients with atrial fibrillation: a report of the American College of Cardiology Foundation/ American Heart Association Task Force on Practice Guidelines developed in partnership with the European Society of Cardiology and in collaboration with the European Heart Rhythm Association and the Heart Rhythm Society. J Am Coll Cardiol 2011; 57: e10198.

15. Shah M, Avgil Tsadok M, Jackevicius CA, Essebag V, Behlouli H, Pilote L. Relation of digoxin use in atrial fibrillation and the risk of all-cause mortality in patients $\geq 65$ years of age with versus without heart failure. Am J Cardiol 2014; 114: 401-6.

16. Sasaki N, Watanabe I, Kogawa R, et al. Effects of intravenous amiodarone and ibutilide on action potential duration and atrial conduction kinetics in patients with persistent atrial fibrillation. Int Heart J 2014; 55: 244-8.

17. Sum CY, Yacobi A, Kartzinel R, Stampfli H, Davis CS, Lai CM. Kinetics of esmolol, an ultra-short-acting beta blocker, and of its major metabolite. Clin Pharmacol Ther 1983; 34: 427-34.

18. Iguchi $\mathrm{S}$, Iwamura $\mathrm{H}$, Nishizaki $\mathrm{M}$, et al. Development of a highly cardioselective ultra short-acting beta-blocker, ONO-1101. Chem Pharm Bull (Tokyo) 1992; 40: 1462-9.

19. Sugiura S, Hidaka K, Seki S, Tsuchida H. Comparative study of two beta adrenergic antagonists on hemodynamics at the endotracheal intubation. Annual meeting of the American Society of Anesthesiologists; 2007: A309. Available at : http://www.asaabstracts. $\mathrm{com} /$ strands/asaabstracts/abstract.htm;jsessionid $=7 \mathrm{AB} 1 \mathrm{~B} 76 \mathrm{~F} 7 \mathrm{CE}$ E0435C8B2198FF104CA4F? year $=2007 \&$ inde $x=4 \&$ absnum $=$ 1298 Accessed Jun 10, 2015.

20. Stevenson LW, Pagani FD, Young JB, et al. INTERMACS profiles of advanced heart failure: the current picture. J Heart Lung Transplant 2009; 28: 535-41. 